\title{
Computational studies of resonance wave pumping in compliant tubes
}

\author{
IDIT AVRAHAMI AND MORTEZA GHARIB ${ }^{2}$ \\ ${ }^{1}$ Medical Engineering, AFEKA-Tel Aviv. Academic College of Engineering, Bney Efraim, \\ Tel Aviv 69107, Israel \\ ${ }^{2}$ Aeronautics and Bioengineering, California Institute of Technology, Pasadena, CA 91125, USA
}

(Received 10 April 2006 and in revised form 6 April 2008)

The valveless impedance pump is a simple design that allows the producion or amplification of a flow without the requirement for valves or impellers. It is based on fluid-filled flexible tubing, connected to tubing of different impedances. Pumping is achieved by a periodic excitation at an off-centre position relative to the tube ends. This paper presents a comprehensive study of the fluid and structural dynamics in an impedance pump model using numerical simulations. An axisymmetric finiteelement model of both the fluid and solid domains is used with direct coupling at the interface. By examining a wide range of parameters, the pump's resonance nature is described and the concept of resonance wave pumping is discussed. The main driving mechanism of the flow in the tube is the reflection of waves at the tube boundary and the wave dynamics in the passive tube. This concept is supported by three different analyses: (i) time-dependent pressure and flow wave dynamics along the tube, (ii) calculations of pressure-flow loop areas along the passive tube for a description of energy conversion, and (iii) an integral description of total work done by the pump on the fluid. It is shown that at some frequencies, the energy given to the system by the excitation is converted by the elastic tube to kinetic energy at the tube outlet, resulting in an efficient pumping mechanism and thus significantly higher flow rate. It is also shown that pumping can be achieved with any impedance mismatch at one boundary and that the outlet configuration does not necessarily need to be a tube.

\section{Introduction}

The principle of an impedance-based valveless pump can be demonstrated by a flexible tube filled with fluid and connected at its ends to tubing of different impedances. By complete or partial pinching of the flexible tube periodically at an off-centre position relative to its ends, a complex series of waves is developed. These waves travel along the tube and reflect at the wave reflection sites. As a result of these wave dynamics, a net flow in a specific direction is observed. Unlike in peristaltic pumps, the direction of the net flow and its magnitude nonlinearly depend on the pinching frequency and duty cycle (Jung \& Peskin 2001; Hickerson, Rinderknecht \& Gharib 2005; Hickerson \& Gharib 2006).

The impedance pump is a simple design that offers a promising new technique for producing or amplifying a net flow for both macro- and micro-scale devices (Rinderknecht, Hickerson \& Gharib 2005), without the requirement for valves or impellers. Moreover, for specific pumping conditions (e.g. pinching frequency, amplitude, 
and duty cycle) the impedance pump may exhibit higher efficiency than a peristaltic pump (Hickerson et al. 2005).

The concept of valveless pumping produced by frequent pinching of compliant tubes has been studied for decades (Liebau 1954). Several experimental works on the pump behaviour have been reported in closed (Liebau 1955, 1963; Ottesen 2003; Hickerson et al. 2005; Rinderknecht et al. 2005) or open (Liebau 1954; Hickerson et al. 2005; Rinderknecht et al. 2005) systems. Several attempts have been made to explain the physical mechanism that drives a net flow in a specific direction in the impedance pump, using analytical or computational models (Rath \& Teipel 1978; Thomann 1978; Moser et al. 1998; Kenner et al. 2000; Jung \& Peskin 2001; Borzi \& Propst 2003; Ottesen 2003; Auerbach, Moehring \& Moser 2004; Manopoulos, Mathioulakis \& Tsangaris 2006). To make the problem tractable, these models often included simplifications and limiting assumptions, thus limiting the validity of the explanation.

Thomann (1978) introduced a one-dimensional analytical model of a closed loop assuming inviscid flow and significant damping of waves in the flexible part. Though Thomann's model predicted a net flow in a specific direction, it mainly attributed the pumping to inertia effects. Moser et al. (1998) suggested a linear electrical analogue model, assuming a closed loop with two distensible reservoirs. The net flow was attributed to inertia and the impedance difference between the two reservoirs. Using that model, they have predicted relations between the net flow rate and the pinching frequency. However, both Thomann and Moser et al. were unable to predict the resonant behaviour of the pump or to predict net flow in an open system.

The dominant role of the wave dynamics in the pump was first introduced by Jung \& Peskin (2001). They simulated the flow in a two-dimensional valveless pump in a closed loop using the immersed boundary method. They described the flow field and the waves travelling along the elastic tube for several cases. They presented examples of pressure waves along the tube and observed some unique wave characteristics in specific cases, such as standing pressure waves in maximal negative flow and travelling pressure waves in maximal positive flow. Their results showed a strong dependence of the net flow on pinching frequency and amplitude: changes in either amplitude or frequency led to nonlinear changes in flow magnitude or direction. However, this model used relatively low mesh and time step resolution, few cases were studied and the mechanics of the pumping action was not described.

Ottesen (2003) presented a simplified one-dimensional numerical model of a closed loop system using periodic boundary conditions. The results were qualitatively compared with experiments. They implied dependence of the flow on pinching amplitude, location, duty cycle, and tube elasticity, and showed a nonlinear dependence of flow rate upon pinching frequency. However, perhaps due to relatively low damping in their model, their results did not seem to converge to a periodic flow and the flow rates obtained were relatively small in comparison with the amplitude of the oscillations.

An improved one-dimensional model was presented by Manopoulos et al. (2006) that used a higher degree of accuracy and took into account the effects of the hydraulic losses due to the stenosis. This allowed convergence to periodic conditions in all the cases considered, with tube contraction ratios as high as $98.5 \%$. Their results exhibit resonance behavior at frequencies close to the natural frequency. Similar to Jung \& Peskin (2001), they also observed a nonlinear dependence of the flow upon pinching amplitude, as well as on some additional parameters such as tube length and pincher width. In their study they presented an example of both pressure and flow waves along the tube, and reported a phase difference between the pressure and flow 
at the tube edges, with a peak value during compression, and suction during tube release. Yet, relying on previous hypotheses by Kenner et al. (2000), they claimed that the driving mechanism in the pump is mainly attributed to asymmetry in hydraulic losses. This explanation does not clarify the reasons for positive flow, flow in open loops or resonant behavior.

Borzi \& Propst (2003) used a one-dimensional simplified set of equations to demonstrate a valveless pump in an open system. Their results showed that an open loop system could create both net flow and net pressure head. The pump characteristics, model simplifications, boundary conditions and solution factors were discussed, with a specific emphasis on the damping affect in the various models. However, an explanation of the driving mechanism of the pump was not given.

Hickerson \& Gharib (2003) suggested the wave reflection at the tube end as a dominant component in the pump action. In a comprehensive experimental study (Hickerson et al. 2005), they referred to the physical dynamics of the pump and investigated several parameters (pincher location and width, pinching frequency, transmural pressure and loop resistance). They suggested that the main pumping mechanism is the wave dynamics developed along the tube, which interact most efficiently at resonant frequencies.

This concept was further demonstrated in a one-dimensional open loop model (Hickerson \& Gharib 2006) based on interactions of pressure waves along an elastic tube with reflection sites. Using this simple model, they could predict some of the characteristics of impedance pumping, proving the major role of wave reflection/interaction in the behavior of the system. However, this simple model did not incorporate the fluid dynamics in the tube, nor the mass, inertial or viscosity effects, and thus the investigation of pumping mechanisms using that model was limited. An additional observation that was introduced in their study was that the resonant behavior is correlated with the natural frequencies of the tubing system as obtained from the system's impulse response. A similar observation was made later by Manopoulos et al. (2006) in their one-dimensional numerical model.

To motivate a better understanding of the complex flow and wave dynamics in the impedance pump, and to explore the physics of its resonant behavior, this work presents a comprehensive numerical study of the fluid and structure dynamics in the impedance pump. A numerical model of an impedance pump in an open system is presented, including the complete partial differential equations (PDE) governing the axisymmetric problem (the Navier-Stokes and structural dynamics equations), where the fluid and structural domains are fully coupled at the interfaces. Results for a wide range of pumping conditions are described, and the resonant nature of the pump is demonstrated. The wave dynamics for resonant and non-resonant cases are depicted and characterized. The energy regime along the tube is analysed, and the physical mechanisms driving the net flow are discussed.

\section{Methods}

\subsection{The physical, mathematical and numerical models}

The impedance pump is simulated as a straight Latex elastic tube filled with water, connected to equal-pressure rigid reservoirs at its both ends, and subjected to radially contracting pinching at a given frequency. To simplify the simulations, an axisymmetric model is used. The model dimensions and material properties resemble the experimental model reported in Hickerson et al. (2005). The tube length is $L=15.2 \mathrm{~cm}$, with inner diameter $d_{i n t}=1.91 \mathrm{~cm}$ and wall thickness $h=0.08 \mathrm{~cm}$. 


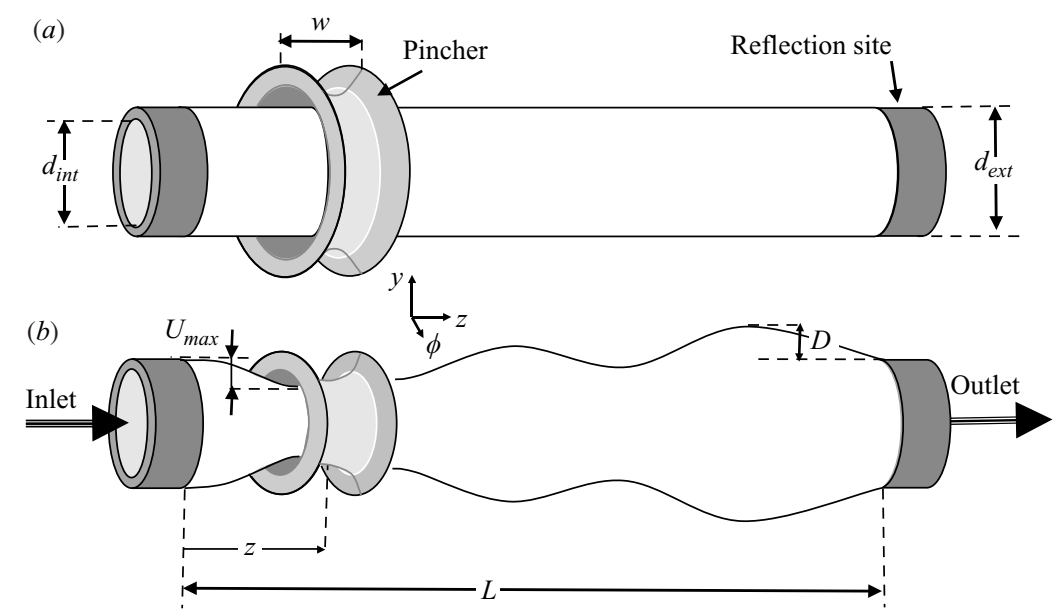

FIGURE 1. An illustrative sketch of the axisymmetric model, at rest $(a)$ and under pinching (b). The illustration presents the elastic tube, the ring-like pincher, the reflection sites, the inlet and the outlet. The following parameters are shown: tube external and internal diameters $\left(d_{\text {ext }}\right.$ and $\left.d_{\text {int }}\right)$, tube length $(L)$, wall displacement from resting diameter $(D)$, pincher location $(z)$, pincher width $(w)$, and maximum pinching $\left(U_{\max }\right)$.

The pincher width is $w=2.54 \mathrm{~cm}$; the Latex elastic tube has elasticity modulus of $E=100 \mathrm{kPa}$, Poisson's ratio of $\nu=0.3$ and density of $\rho_{t}=1 \mathrm{~g} \mathrm{ml}^{-1}$; the fluid was defined as water.

Due to the axisymmetric assumption, the numerical model had a few differences with respect to the experimental model. The radial contraction at pinching does not conserve the tube circumference. This radial pinching is different from the flat pinching in the experiments. However it has been shown in the experiments that the shape of the waves is non-radial only in the vicinity of the pincher and two diameters downstream of the pincher the waves are radial even if the pinching is flat (Hickerson 2005). Thus, this simplification may change the wave velocity, but does not affect the characteristics of the flow and structure in a typical impedance pump.

A finite-element scheme was used to solve the set of motion and fluid equations using the commercial software ADINA (ADINA R\&D Inc., MA). The numerical model (illustrated in figure 1, see also figure 11) included the elastic tube, the pincher and the fluid. The numerical calculations incorporated the contact of the pincher with the elastic wall, the dynamics of the flexible tube, the fluid-structure interaction between the solid and the fluid at the interface, and the dynamics of the fluid.

The dynamics of the flexible wall were calculated using the linear dynamics response of a system:

$$
M \ddot{U}+C \dot{U}+K U=R
$$

where $\boldsymbol{M}, \boldsymbol{C}$, and $\boldsymbol{K}$ are the mass, damping and stiffness matrices; $\boldsymbol{R}$ is the vector of externally applied loads; $\boldsymbol{U}=\left(u_{y}, u_{z}\right)$ is the displacement vector and $\dot{\boldsymbol{U}}$ and $\ddot{\boldsymbol{U}}$ are the velocity and acceleration vectors of the structural domain, respectively (Bathe 1996, p. 672).

The maximal strains of the flexible wall were around $30 \%$, which are in the linear range of the material elasticity (Hickerson 2005), thus large deformations and small strain were considered in the simulations and the stress-strain relationship of the 
viscoelastic material was assumed to be linear:

$$
\sigma=E\left(\varepsilon+\varepsilon_{0}\right)
$$

where $\varepsilon$ and $\sigma$ are the instantaneous strain and stress, respectively, and $\varepsilon_{0}$ is the initial strains

The flow and pressure fields were calculated by solving numerically the equations governing momentum and continuity in the changing fluid domain (moving mesh) (Rosenfeld \& Kwak 1991):

$$
\begin{gathered}
\boldsymbol{\nabla} \cdot \boldsymbol{V}=0, \\
\rho_{w}\left(\frac{\partial \boldsymbol{V}}{\partial t}+\left(\boldsymbol{V}-\boldsymbol{V}_{g}\right) \cdot \nabla \boldsymbol{V}\right)+\nabla p=\mu \nabla^{2} \boldsymbol{V},
\end{gathered}
$$

where $\boldsymbol{V}=\left(v_{y}, v_{z}\right)$ is the flow velocity vector and $\boldsymbol{V}_{g}$ is the velocity vector of the moving local coordinates, $p$ the static pressure, $t$ the time, $\rho_{w}=1 \mathrm{~g} \mathrm{ml}^{-1}$ and $\mu=$ $0.01 \mathrm{~g} \mathrm{~cm}^{-1} \mathrm{~s}^{-1}$ are the density and the dynamic viscosity of water, respectively. The ALE moving mesh approach (Bathe 1996, p. 768) was used to adjust the mesh to the boundary motion.

At the fluid-structure interfaces, displacement compatibility (for no-slip conditions) and traction equilibrium were applied:

$$
\boldsymbol{V}_{f}=\dot{\boldsymbol{U}}_{s}, \quad \boldsymbol{n} \cdot \boldsymbol{\tau}_{f}=\boldsymbol{n} \cdot \boldsymbol{\tau}_{s},
$$

where $\boldsymbol{V}_{f}$ and $\dot{\boldsymbol{U}}_{s}$ are the fluid and solid velocities at the interface, respectively and $\boldsymbol{\tau}_{f}$ and $\tau_{s}$ are the fluid and solid stresses, respectively. The fluid and structure domains were coupled at the interface, using two-way coupling (simultaneous solution method), where the fluid equations and the solid equations are combined and treated as one complete system of linear equations (Rugonyi \& Bathe 2001).

The pincher was modelled as a moving rigid surface with prescribed harmonic motion dictated by the excitation parameters of each case: pinching frequency $(f)$, pinching amplitude ( $A$, the ratio between the maximal pinching $U_{\max }$ and the tube radius $R$ ) and duty cycle $(D C$, the ratio between the duration of active pinching and the cycle period). The time-dependent pincher location $U(t)$ was determined by:

$$
U(t)=\beta+R+\alpha \sin [2 \pi f(t-\phi)]
$$

where $t$ is time, and $\delta, \beta$ and $\phi$ are mathematical constants defined as:

$$
\left.\begin{array}{l}
U_{\max }=A R, \\
\delta=\frac{U_{\max }}{1-\sin (\pi(0.5-D C)}, \\
\beta=U_{\max }-\delta, \\
\phi=\frac{2 D C-1}{4 f} .
\end{array}\right\}
$$

Once the moving pincher crossed the outer surface of the tube wall, ADINA's contact analysis was employed using the Constraint-Function method (Bathe 1996, pp. 625628; ADINA R\&D 2005).

In order to model an elastic tube connected to two equal-pressure rigid reservoirs, similar to the experimental model (Hickerson et al. 2005), the tube ends were fixed and zero pressure conditions were employed at both inlet and outlet. The rigid connections were simulated by fixing both ends of the elastic tube. This was carried 
out by imposing zero displacement $u_{y}=u_{z}=0$ on the nodes at the two edges of the solid model, resulting in full reflection of the elastic waves. The fluid at the tube ends was subjected to zero normal traction boundary conditions. The normal traction is defined as the normal stress $\left(\tau_{n n}=\boldsymbol{n} \cdot \boldsymbol{\tau} \cdot \boldsymbol{n}\right)$ which consists of the pressure and the normal shear stress ( $\boldsymbol{\tau}$ being shear stress tensor $\boldsymbol{n}$, the normal to the boundary). Since the prescribed pressure at the outlet was assumed constantly zero, these conditions resulted in stress-free conditions at both ends (ADINA R\&D 2005, p. 90).

The fluid and solid domains were meshed with 4-noded axisymmetric elements. The mesh was refined in both the solid and fluid domain around the excitation zone. According to mesh and time-step independence tests (for working conditions of $z=2.54 \mathrm{~cm}, A=80 \%, D C=20 \%, f=4 \mathrm{~Hz}$, where these parameters will be defined later), a model with a total of 8000 elements and 1000 steps per cycle was found to give average accuracy within $2 \%$. All simulations began at a state of rest both at the fluid and solid domains $(\boldsymbol{U}=0, \boldsymbol{V}=0, D=0, p=0)$. Each calculation continued for 30 pumping cycles, when a complete steady periodic state was always achieved.

\section{Results}

\subsection{Wave speed and natural frequency}

First, the inherent properties of the model were calculated. The simulated speed of a single pressure wave propagating along the tube was $180 \mathrm{~cm} \mathrm{~s}^{-1}$. This value agrees with the approximated Moen-Korteweg formula for waves in a fluid field elastic tube (Zamir 2000):

$$
c_{0}=\sqrt{\frac{E h}{\rho_{w} d}} \approx 200 \mathrm{~cm} \mathrm{~s}^{-1} .
$$

The response of the system to impulse pinching was examined by following the dynamics of the pump as a response to a single pinch for different pinching locations. In each case, a single short pinching of radius contraction at $A=80 \%$ was imposed for $0.02 \mathrm{~s}$ and then the model was released to oscillate at the system's natural frequency for $4.5 \mathrm{~s}$, with sampling intervals of $0.002 \mathrm{~s}$. The same natural frequency of $6.12 \mathrm{~Hz}$ and its harmonics at $12.23 \mathrm{~Hz}$ and $18.09 \mathrm{~Hz}$ were found for different pinching locations, for different sampling locations and for different parameters (flow, pressure and wall displacement). For example, the time-dependent wall displacement and its spectral density are given in figure 2. This natural frequency is equivalent to the rate at which a single wave would travel back and forth the length of the tube at a speed of $186 \mathrm{~cm} \mathrm{~s}^{-1}$.

\subsection{Bulk flow rate and transient response}

An algorithm for flow rate calculation at the distal outlet (marked 'outlet' in figure 1) was developed by integrating the velocity profiles over the outlet elements. Flow rates are considered positive when flow exits the distal end. A typical transient response of flow rate is shown in figure 3. As shown by the plot, there is a transitional stage for the flow to build up (rise time) before the average flow reaches its steady-state mean. After reaching a steady periodic state, the average flow rate in a pinching period is an indication of the bulk flow of the pump for the specific pumping condition. The complex dynamics of reflecting and interacting waves in each case results in a different number of cycles in the build-up. The rise time is defined as the number of cycles required for the bulk flow rate to reach $98 \%$ of its final level. 

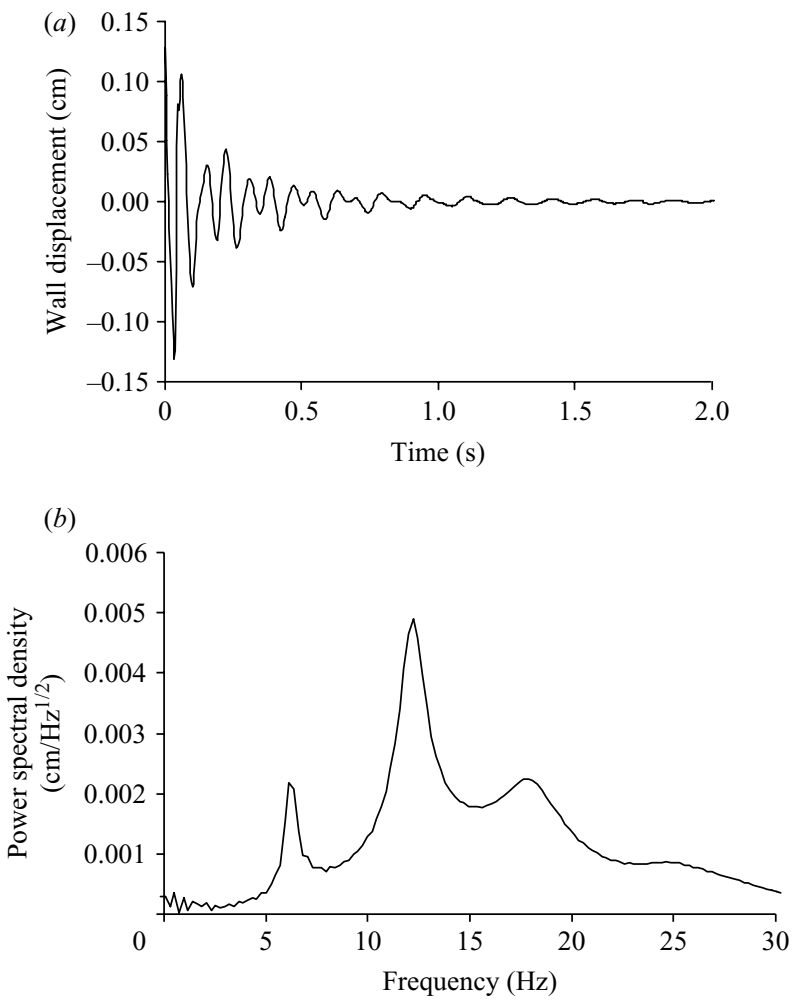

FIGURE 2. System's response to impulse pinching: $(a)$ the transient response of wall displacement of the elastic tube $3 \mathrm{~cm}$ downstream the pincher; $(b)$ power spectrum density (using fast fourier transform) of the signal, indicating a natural frequency at $6.12 \mathrm{~Hz}$ and harmonics at $12.23 \mathrm{~Hz}$ and $18.09 \mathrm{~Hz}$.

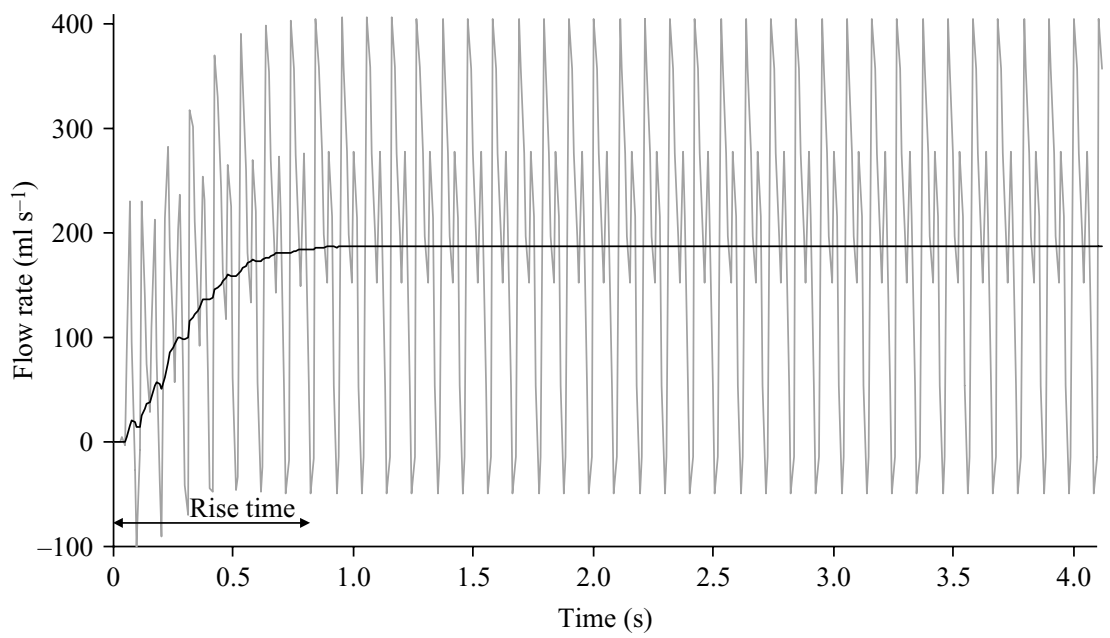

Figure 3. A typical transient response of the outlet flow rate. The solid line is a filtered curve of the flow rate using a moving average window of one cycle. The average flow rate of the periodic phase in this case $(9.5 \mathrm{~Hz})$ is $187 \mathrm{ml} \mathrm{s}^{-1}$. 

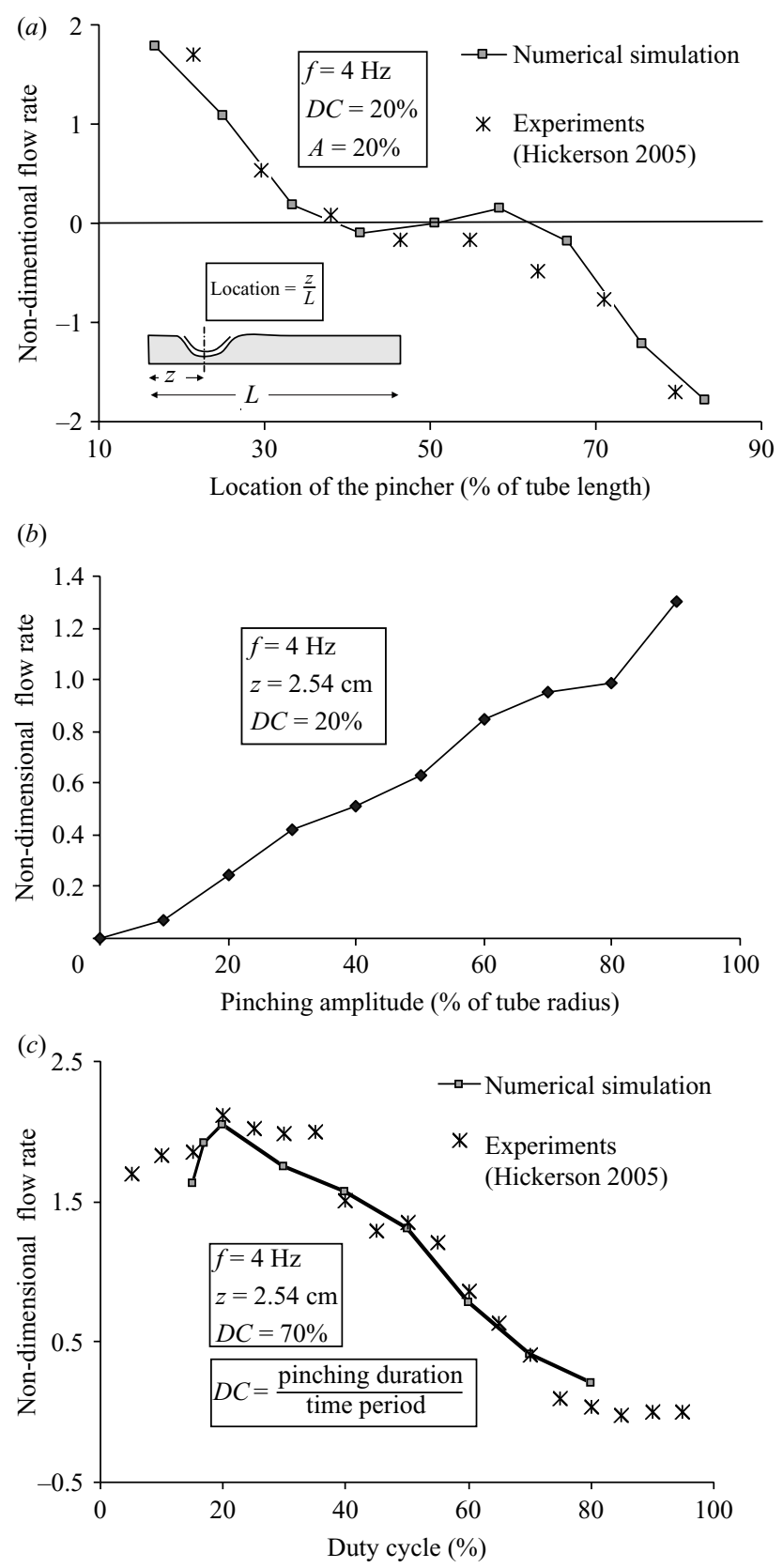

FIGURE 4. Non-dimensional flow rate as calculated from the simulations, and as measured experimentally by Hickerson (2005) as a function of $(a)$ pinching location along the tube, $(b)$ pinching amplitude, and $(c)$ pinching duty cycle.

\subsection{Effect of pinching parameters}

In the second stage, the effects of the pinching location, amplitude, duty cycle, and frequency on the outlet bulk flow were examined. The calculated bulk flow as a function of these parameters is shown in figure 4 and figure 5. For comparison with experimental results, the flow rate in figure 4 is non-dimensionalized by half the rate 
of volume displaced by the pinchers as suggested by Thomann (1978) and Hickerson et al. (2005).

\section{Pinching location}

The pinching location (defined as $z$ in figure 1) is presented in figure $4(a)$ as a percentage of the total length of the elastic tube $(L)$. As the location of the pincher shifts towards the centre, the bulk flow rate reduces to a negligible level. Shifting of the pincher further towards the outlet end resulted in negative net flow behaving symmetrically. These results are in agreement with experimental data collected by Hickerson (2005). The negligible non-zero flow rate around the 50\% location in Hickerson's experimental results was not addressed by the author, and may be the result of a cumulative affect in the calculation of average net flow from several cycle measurements.

\section{Pinching amplitude}

For the fixed pinching frequency $f=4 \mathrm{~Hz}$ and $z=2.54 \mathrm{~cm}$ (location $=16.7 \%$ ), the flow increases with pinching amplitude $A$ (figure $4 b$ ). These results are in agreement with Hickerson et al. (2005), and are somewhat different from results in closed loops (Jung \& Peskin 2001; Manopoulos et al. 2006) which both found nonlinear correlation between the flow and the pinching amplitude.

\section{Duty cycle}

In the present work, the duty cycle $(D C)$ was defined as the ratio between the duration of active pinching and the cycle period. figure 4(c) shows that in the cases simulated, larger bulk flow is obtained for smaller duty cycles. These results also agree with the experiments reported by Hickerson (2005). (Note the different definition of $D C$ in the reference.)

\section{Pinching frequency}

To examine effect of pinching frequency, a series of simulations were performed to scan the pinching frequency range $2-20 \mathrm{~Hz}$. In order to optimize computational effort, non-uniform frequency test intervals were used. Higher resolution tests were conducted in ranges of interest. The corresponding non-dimensional Womersley range as suggested by Hickerson et al. (2005) for these frequencies is 13.5-40, where Womersley number is defined as $\alpha=\sqrt{\rho f / \mu}$. However, such non-dimensional generalization should be considered with care, since it might exclude non-linear factors in the structural system (the elastic tube) which cannot be non-dimensional using Womersley's number. Therefore, in this case, the dimensional results are given.

In all the frequency-test simulations, the other parameters $A=70 \%, D C=20 \%$ and $z=2.54 \mathrm{~cm}$ were kept constant. The results are shown in figure 5 . Higher bulk flow was obtained for frequencies $5.9 \mathrm{~Hz}(\alpha=23)$ and $11.5 \mathrm{~Hz}(\alpha=32.2)$. These results correspond to the model's natural frequency of $6.12 \mathrm{~Hz}$ and its harmonics, thus agreeing with the hypothesis of a resonant mode. A comparison with the experiments was conducted for similar conditions $(D C=20 \%, z=2.54 \mathrm{~cm}, A=70 \%)$. There were data only for frequency range $f=1-10 \mathrm{~Hz}$, where the low computational resonance frequency $(f=5.9 \mathrm{~Hz})$ correlates well with the experimental resonance frequency $f=6.3 \mathrm{~Hz}$. The differences in flow rate values are attributed to the different pinching method (flat pinching versus ring-like pinching) and the connected tubing (closed loop versus open loop). 


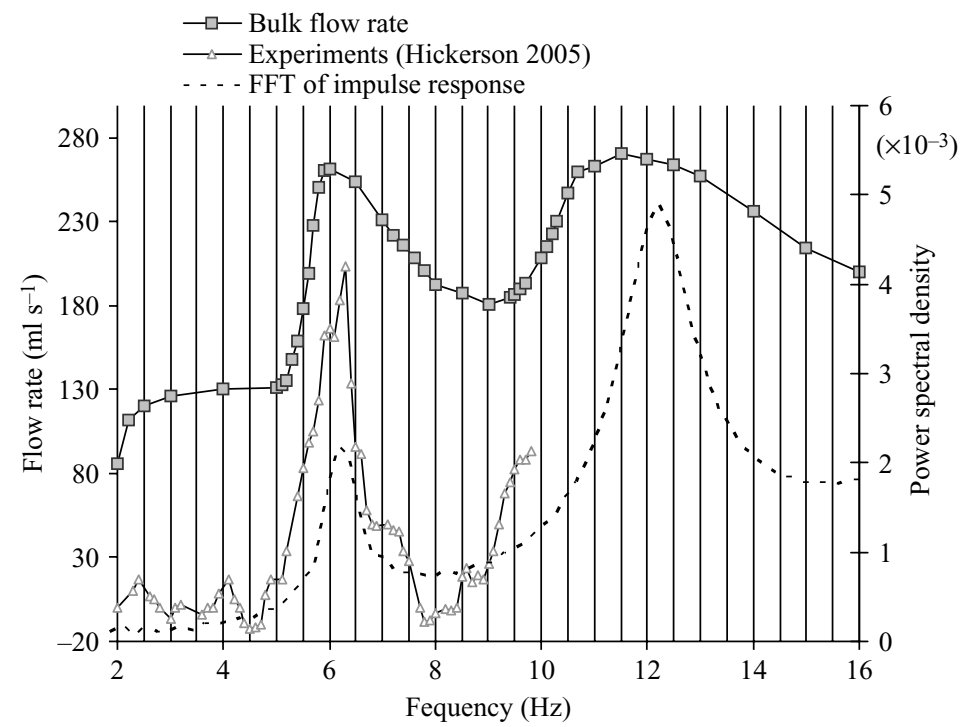

FIGURE 5. Net flow rate as a function of pinching frequency (dark line), for pinching amplitude of $70 \%$ and duty cycle of $20 \%$. The results are compared with the spectral density of the impulse response (dashed line) and the experimental measurements of Hickerson (2005) (light line).
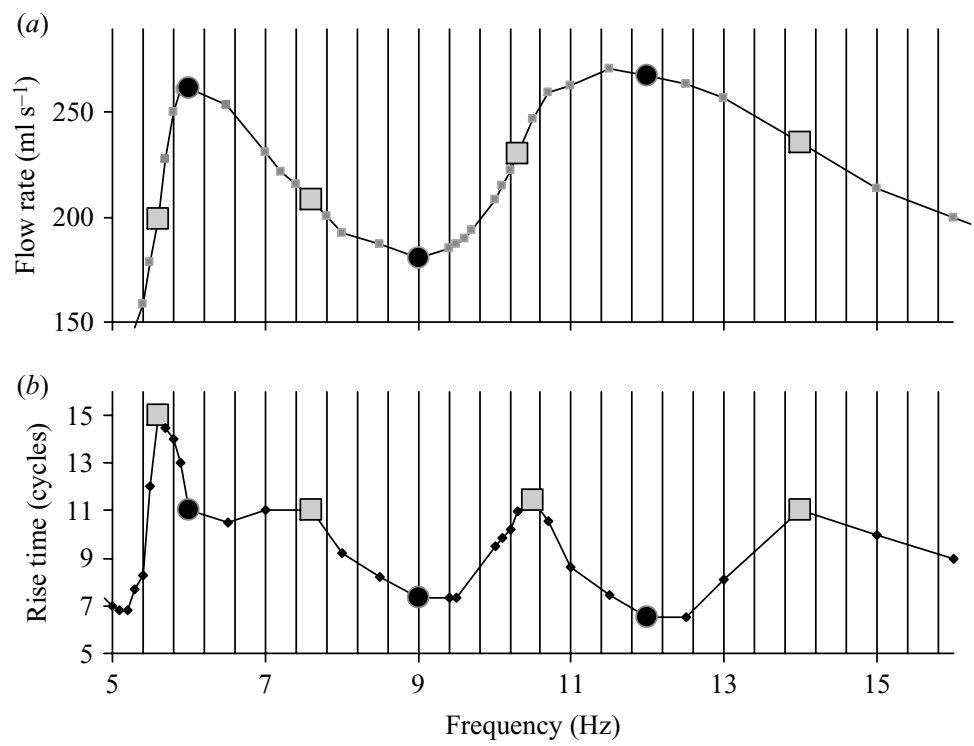

FIgURE 6. Rise time (in cycles) for frequencies range $5-16 \mathrm{~Hz}(b)$ is compared with the magnitude of the bulk flow $(a)$. The cases with large and small rise times are marked with squares and circles, respectively.

\subsection{System rise time}

Figure 6 depicts the rise times, defined in figure 3, measured in number of cycles, for all the simulated cases in the frequency range $2-16 \mathrm{~Hz}$ (black line). The rise time plot is compared with the bulk flow rate magnitude. The shortest rise times are found 


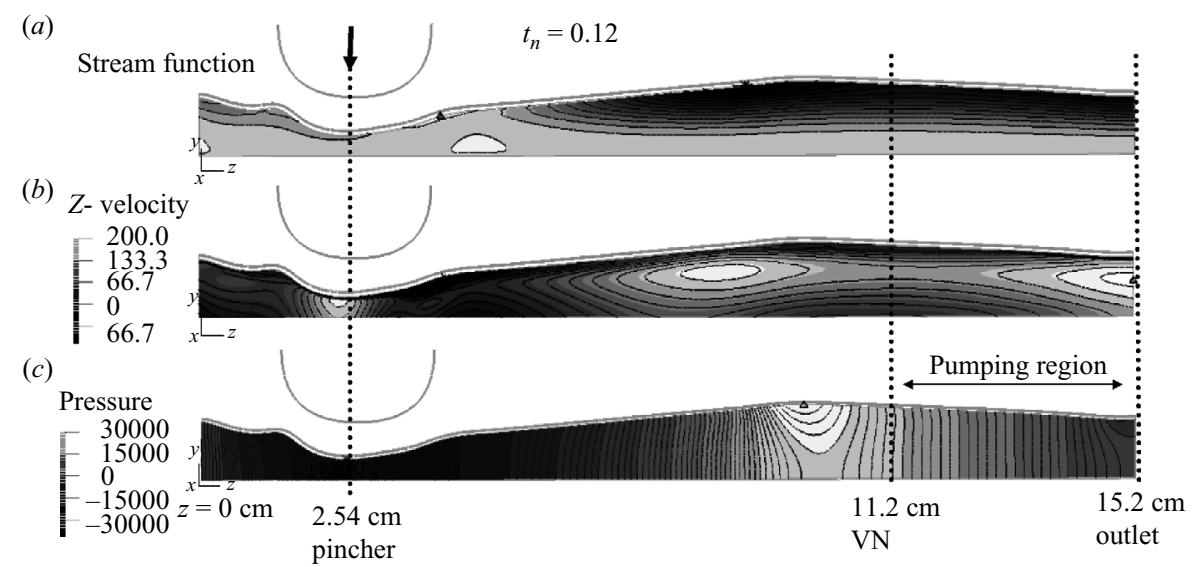

Figure 7. An example of the simulated flow field of the high-flow case $11.5 \mathrm{~Hz}$. Streamlines, pressure and $z$-velocity plots (bottom) at. $t_{n}=0.12$. The location of the pincher, the velocity node (VN) and the outlet cross-section are indicated by dotted lines. The 'pumping region' downstream of the velocity node is also indicated.

at frequencies with extremely high flow rates $(f=6$ and $12.5 \mathrm{~Hz})$ or extremely low flow rates $(f=9 \mathrm{~Hz})$ (marked with circles on the plots), while longest rise times are found at 'transitional' frequencies $(f=5.6,7.6,10.3$, and $14 \mathrm{~Hz})$ which lie on the slopes between high and low flow rates (marked with squares). These results are in agreement with some qualitative observations reported by Hickerson (2005) and indicate the complex and delicate balance between constructive and destructive wave interactions taking place in the steady periodic flow field. In the extreme cases, the wave dynamics is led by a dominant wave system (either constructive or destructive), and thus converge faster to steady state. In the transitional cases, on the other hand, the waves dynamic is less clear, and thus it requires more waves to participate in the complex waves system until the flow converges to periodic conditions.

\subsection{Waves dynamics along the tube}

From the numerical simulation, a full description of the time-dependent fluid and structure fields is given, as shown for example in figure 7. In addition, in figure 7 the locations of three specific cross-sections along the tube to be referred later are indicated: the pincher, the velocity node $(\mathrm{VN})$ and the outlet cross-section, and the 'pumping region' between the VN and the outlet.

Figure 8 shows waves envelopes formed from flow rate, pressure and wall displacements along the tube at different times within the periodic cycle, for seven typical cases. The wall displacement $(D)$ is measured from the tube resting radius. In all the envelopes formed, wave characteristics are observed including nodes and antinodes, where nodes are points where the parameter variations are minimal, and antinodes are points that undergo large variations (Nichols \& O'Rourke 1998).

Downstream of the pincher, there is a strong correlation between pressure and wall displacement due to the linearly elastic properties of the tube wall and the relatively uniform distribution of pressure in the tube cross-section. The flow envelopes behave differently from the pressure due to the phase lag between velocity and pressure in the elastic tube. In all the cases a 'velocity node' (VN) is observed downstream of the tube, in the vicinity of a pressure antinodes. The axial location of the $\mathrm{VN}$ is different for each case and is marked on the plots by dashed lines. 


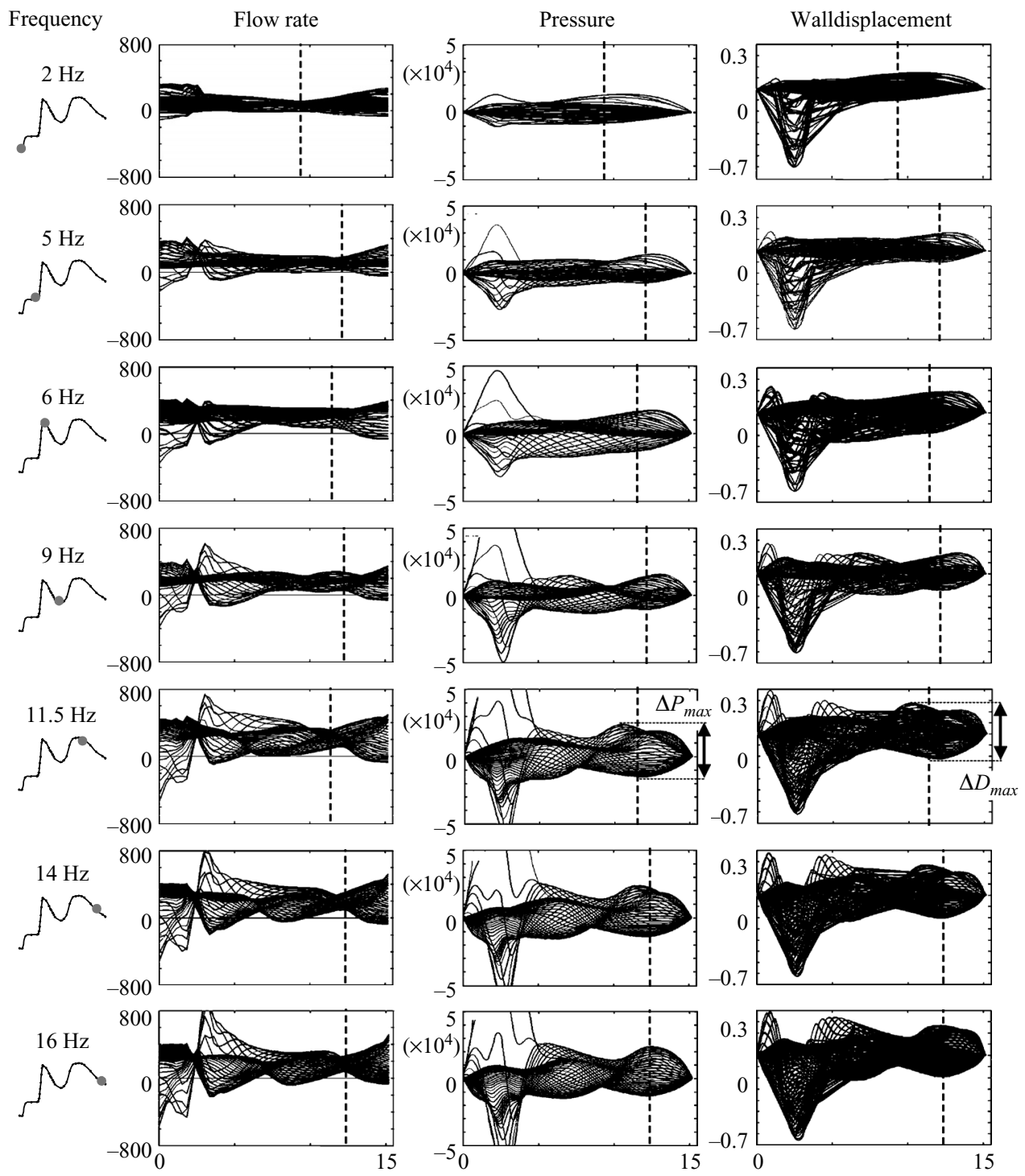

FiguRE 8. Progression of flow (left), pressure (middle) and wall displacement (right) along the tube, plotted using individual distribution curves at different times during the periodic cycle for representative cases. The marks on the frequency plots represent the net-flow rate of the corresponding case on the flow-frequency plot. The locations of the VN of each case are indicated by dashed lines.

The largest variations of pressure and radial displacement are found near the VN of the high flow case $(11.5 \mathrm{~Hz})$, as marked in figure 8 , with $\Delta P_{\max }=42500 \mathrm{dyn} \mathrm{cm}^{-2}$ $(4.25 \mathrm{kPa})$ and $\Delta D_{\max }=0.5 \mathrm{~cm}$. As will be shown later, these VN also have special significance for the relationship between elastic and kinetic energy along the tube.

\subsection{Pressure and velocity fields}

Figure 9 shows plots of the time-dependent flow and pressure fields along the tube for nine time sequences during a pinching cycle of the resonant case, $f=11.5 \mathrm{~Hz}$. As mentioned before, the pressure downstream of the pincher is correlated to the tube 


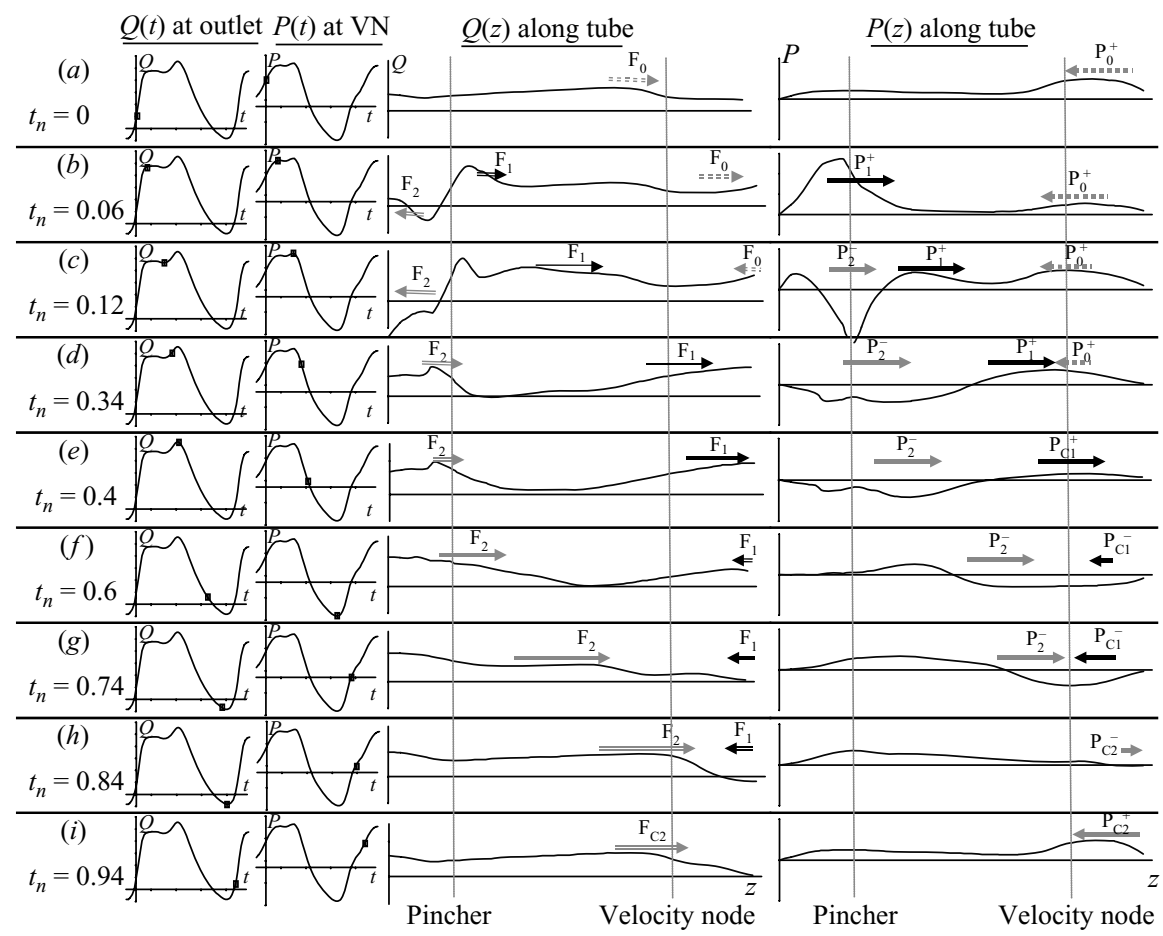

FIGURE 9. Flow and pressure in the model shown for nine instances along a typical pinching cycle of the high-flow case $(11.5 \mathrm{~Hz})$. At the left the normalized time is indicated for each instance, and is marked by a point on the time-dependent plot of outlet flow (second column) and pressure at the velocity node (third column). In the fourth and fifth columns, flow and pressure are shown along the tube. The single and double line arrows on the plots indicate pressure and flow waves, respectively: dark arrows indicate waves originated by the pinching and light arrows indicate waves originated by restoring forces. Dashed arrows indicate waves that were originated in the previous cycle.

with radius. Time is indicated by normalized time $t_{n}=t f$. Each sequence is indicated by a dot on the plots of the time-dependent flow at the outlet, and the pressure at the VN. Pressure and flow waves travelling along the tube are indicated by arrows and denoted by 'P' and ' $\mathrm{F}$ ' with subscript numbers respectively. Below, six stages along the cycle are detailed, describing the periodic wave dynamics in the tube.

\section{Stage 1: Just before pinching (figure 9a)}

The cycle starts just before pinching, with a forward flow pulse $\left(\mathrm{F}_{0}\right)$ that originated in the previous cycle travelling downstream towards the outlet and a high-pressure wave that $\left(\mathrm{P}_{0}^{+}\right)$travels backwards upstream. At that instant both the time-dependent outflow and VN pressure approach local maxima.

\section{Stage 2: Pinching and releasing (figure $9 b, c$ )}

As a result of the pinching, a forward flow pulse $\left(F_{1}\right)$ and a high-pressure wave $\left(\mathrm{P}_{1}^{+}\right)$are formed and travel downstream in the tube. As the pincher is released, a low-pressure wave that is a result of restoring forces at the pinching location $\left(\mathrm{P}_{2}^{-}\right)$is formed and travels downstream as well. This wave is followed by a second flow pulse $\left(\mathrm{F}_{2}\right)$ emerging from the tube inlet. 
Stage 3: $\mathrm{P}^{+}$wave interaction - tube expansion (figure 9d,e)

At $t_{n}=0.34$, the pressure wave $\left(\mathrm{P}_{1}^{+}\right)$interacts with the backward high-pressure wave $\left(\mathrm{P}_{0}^{+}\right)$near the VN. The interaction of the two high-pressure waves $\left(\mathrm{P}_{1}^{+}\right.$and $\left.\mathrm{P}_{0}^{+}\right)$results in a combined high-pressure wave $\left(\mathrm{P}_{\mathrm{C} 1}^{+}\right)$. Since the forward wave $\left(\mathrm{P}_{1}^{+}\right)$is stronger than the backward wave $\left(\mathrm{P}_{0}^{+}\right)$, the resulting wave $\left(\mathrm{P}_{\mathrm{C1}}^{+}\right)$travels downstream with decreased amplitude. Consequently, the time-dependent VN pressure reaches a maximum. As a result of the increased VN pressure, the tube is locally expanded and produces a volume suction of fluid to fill the cavity. This suction draws and enhances the flow pulse $F_{1}$ that reaches the tube end and thus increasing the time-dependent outlet flow.

\section{Stage 4: waves reflections (figure $9 f$ )}

The combined pressure wave $\left(\mathrm{P}_{\mathrm{C} 1}^{+}\right)$reaches the tube end at $t_{n}=0.4$ and is reflected by the reflection site. On reflection, both its sign and direction are reversed, thus it starts travelling backwards (upstream) as a low-pressure wave $\left(\mathrm{P}_{\mathrm{C} 1}^{-}\right)$. At this stage, the flow pulse $\left(F_{1}\right)$ is reflected at the tube end as well, and the time-dependent outlet flow starts decreasing.

Stage 5: $\mathrm{P}^{-}$wave interaction - tube compression (figure $9 f, g$ )

At $t_{n}=0.74$, the low-pressure wave $\left(\mathrm{P}_{2}^{-}\right)$reaches the $\mathrm{VN}$, where it meets the backward combined wave $\left(\mathrm{P}_{\mathrm{C} 1}^{-}\right)$. The interaction of the two low-pressure waves near the $\mathrm{VN}$ results in a minimum pressure and rapid tube compression. Since $\mathrm{P}_{2}^{-}$is stronger than $\mathrm{P}_{\mathrm{C} 1}^{-}$, the combined pressure wave $\left(\mathrm{P}_{\mathrm{C} 2}^{-}\right)$travels downstream.

Stage 6: $\mathrm{P}^{-}$wave reflection (figure $9 h, i$ )

As it reaches the tube end at $t_{n}=0.84$, the combined low-pressure wave $\left(\mathrm{P}_{\mathrm{C} 2}^{-}\right)$ is reflected by the tube end and travels backwards as a high-pressure wave $\left(\mathrm{P}_{\mathrm{C} 2}^{+}\right)$. Consequently, the tube is rapidly expanded at the $\mathrm{VN}$, resulting in strong volume suction. In order to fill the expanded tube, the backward flow $F_{1}$ and forward flow $F_{2}$ propagate towards the VN. Since $F_{2}$ is much stronger than $F_{1}$, the combined wave $\mathrm{F}_{\mathrm{C} 2}$ is positive (towards the outlet). After passing the high-pressure region (of $\mathrm{P}_{\mathrm{C} 2}^{+}$), the flow $F_{C 2}$ is washed out by the pressure gradient. As a result, the time-dependent outlet flow undergoes a rapid increase. At this stage a new cycle begins, while the waves $\mathrm{F}_{\mathrm{C} 2}$ and $\mathrm{P}_{\mathrm{C} 2}^{+}$are $\mathrm{F}_{0}$ and $\mathrm{P}_{0}^{+}$of the next cycle, respectively.

\subsection{P-Q loops as indication of energy transform}

The time-dependent pressure and flow of the high-flow case were extracted at three points in the vicinity of the VN $(z=10.42,11.21$ and $12 \mathrm{~cm})$. Figure 10 shows the periodic pressure and flow at these points plotted as a function of time, and in pressure-flow loops ( $P-Q$ loops). The $P-Q$ loops are marked by dark and light arrows indicating loop direction. Dark arrows indicate clockwise loop direction, implying cases in which the flow follows the pressure. Light arrows indicate a counterclockwise loop direction, implying cases in which flow precedes pressure.

The physical significance of $P-Q$ loop area is power (energy per cycle period), where clockwise (dark arrows) loops represent power given to the fluid by the elastic tube, and counterclockwise loops (light arrows) represent conversion power given to the elastic tube by the fluid. In this analysis, the energy of the fluid in the tube consists of kinetic and potential energy and will be referred to henceforth as 'fluid energy' (to distinguish it from 'elastic energy'). 

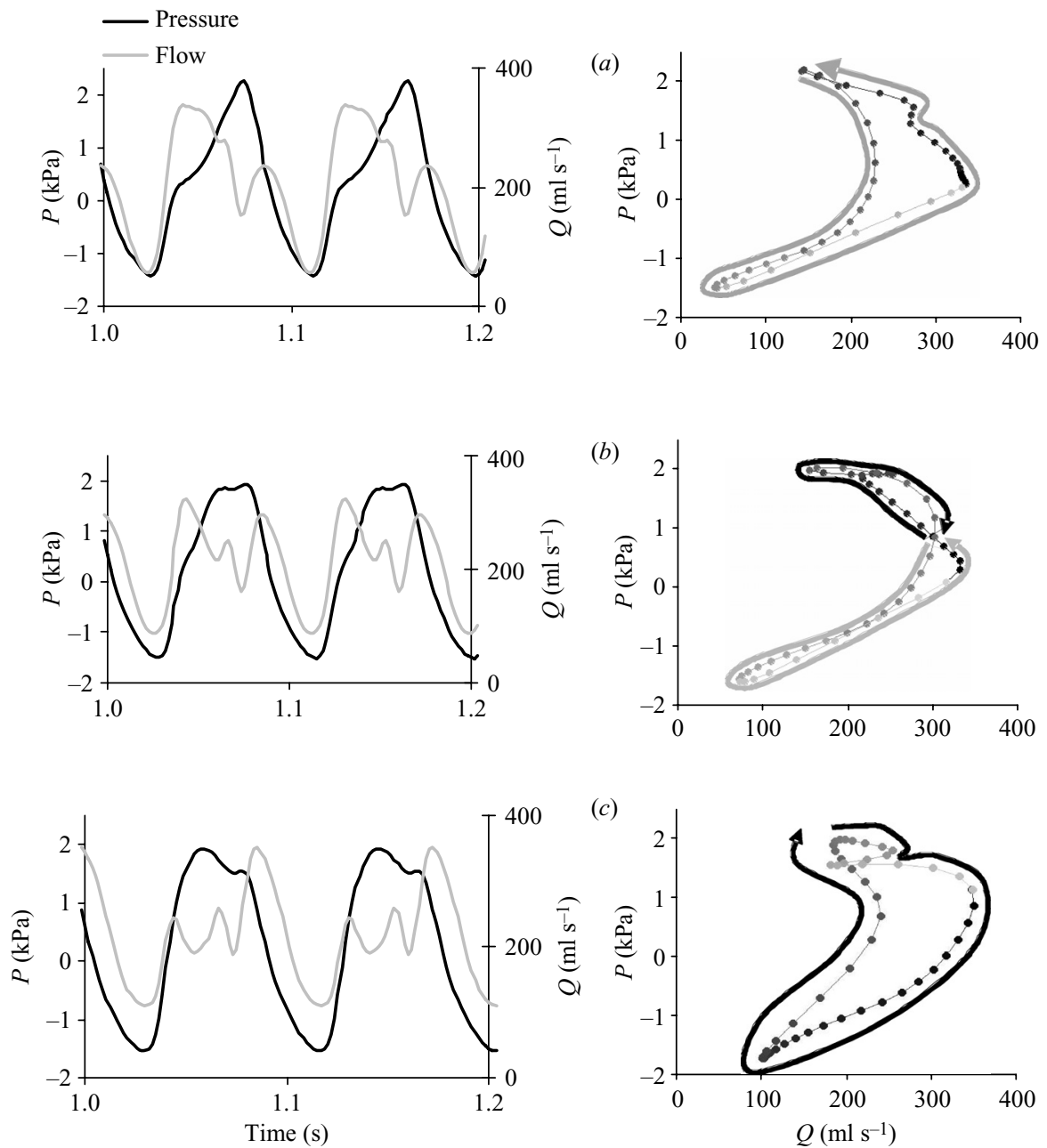

Figure 10. Pressure and flow rate as a function of time (left), and pressure-flow loops (right) at three points in the vicinity of the velocity node for $11.5 \mathrm{~Hz}$. Dark arrows indicate clockwise loop direction, and light arrows indicate counterclockwise loop direction. $(a) z=10.5 \mathrm{~cm},(b)$ $z=11.2 \mathrm{~cm},(c) z=12 \mathrm{~cm}$.

To obtain the power given to the fluid at each point, the total area of each loop was calculated, considering clockwise loops as positive and counterclockwise loops as negative. The loops change direction on passing through the $\mathrm{VN}$, where at the $\mathrm{VN}$ (at $z=11.21 \mathrm{~cm}$ ) the area of the $P-Q$ loop collapses to minimum.

Similar $P-Q$ loops are plotted in figure 11 at seven points downstream in the tube for three representative frequencies (low-flow cases: 9 and $14 \mathrm{~Hz}$ and high-flow case $11.5 \mathrm{~Hz}$ ). The sampling points $1-7$ are distributed evenly along the long passive region of the tube between $z=4 \mathrm{~cm}$ and $z=14 \mathrm{~cm}$. Point 1 indicates the power given to the fluid by the pincher. Point 7 indicates the energy given to the fluid at the outlet (i.e. total resulting power of the pump). The $\mathrm{VN}$ of the $11.5 \mathrm{~Hz}$ case is located between points 5 and 6 . The $P-Q$ loops shown have the same scale as figure 10 and are shaded according to their direction, as specified above (dark for clockwise and light for counterclockwise). 

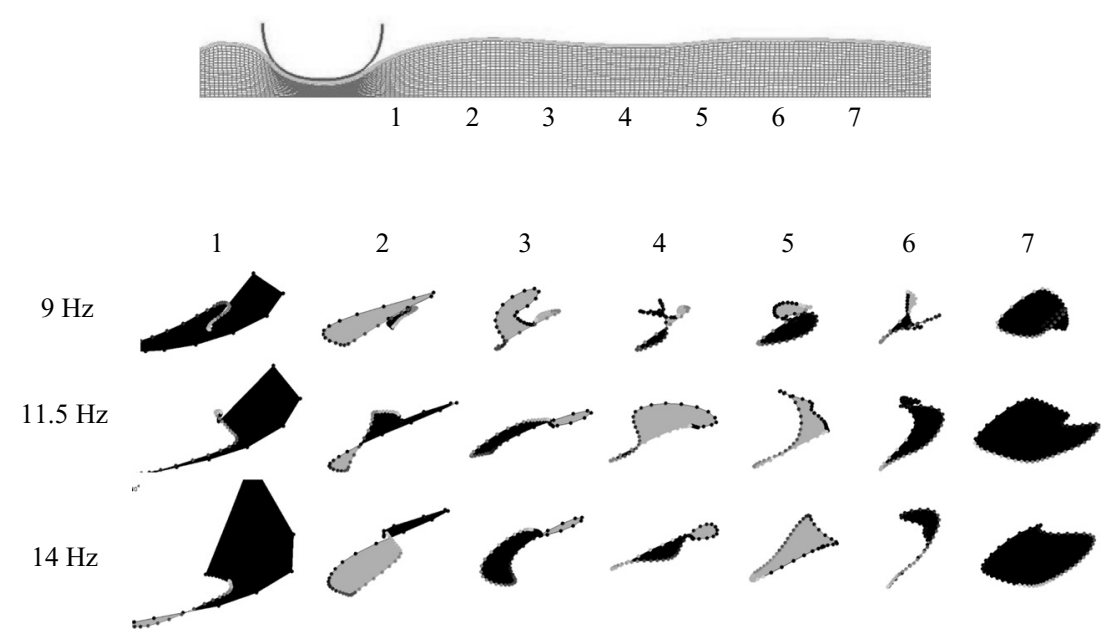

FIGURE 11. Pressure-flow loops taken at seven points along the tubes for representative cases $(9,11.5,14 \mathrm{~Hz})$ (areas filled with dark shading indicate clockwise loop direction and areas filled with light shading indicate counterclockwise loop direction).

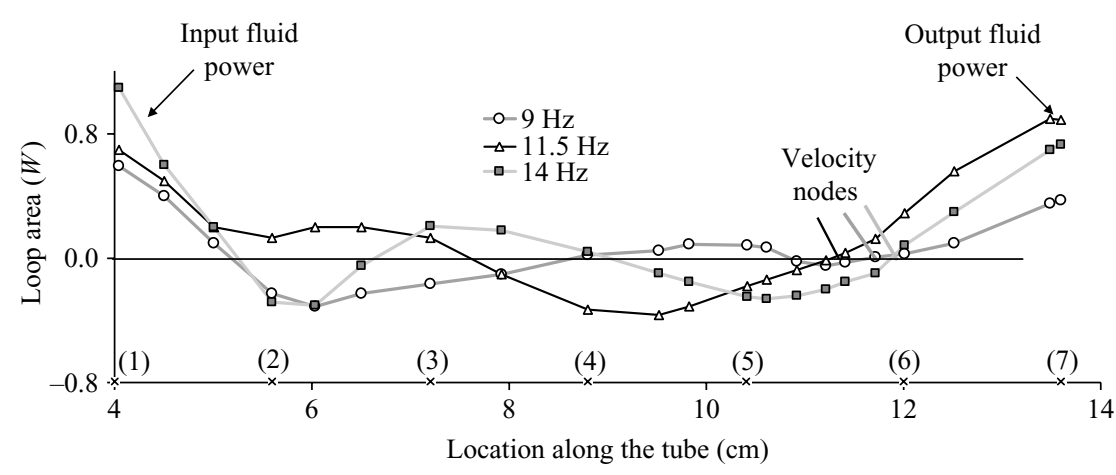

Figure 12. P-Q Loops areas for 9, 11.5 and $14 \mathrm{~Hz}$ as calculated at 21 points along the passive tube (points 1-7 are marked for reference with figure 11).

Loop areas of the above three representative cases as calculated at 21 points along the long passive region are shown as a function of axial location $z$ in figure 12. The areas of the loops are changing along the tube for all the frequencies simulated, where negative areas indicate conversion of fluid energy to elastic energy, and positive areas indicate elastic energy converted to fluid energy. For each case, the loop area changes sign at its $\mathrm{VN}$. That means that the region downstream of the $\mathrm{VN}$ is where the 'pumping' occurs, i.e. conversion of the elastic energy of the tube into fluid energy (see 'pumping region' in figure 7).

\subsection{Calculation of total work done on the fluid}

In order to extract the net power added to the fluid by the elastic tube at each cycle, the accumulated power given to the fluid was calculated for all the cases $f=8-15 \mathrm{~Hz}$ using integration of the P-Q loop area along the passive region $z=4-14 \mathrm{~cm}$ :

$$
\dot{W}_{\text {acc }}=\int_{z=4 \mathrm{~cm}}^{z=14 \mathrm{~cm}}\left(P Q_{\text {area }}\right) \mathrm{d} z
$$



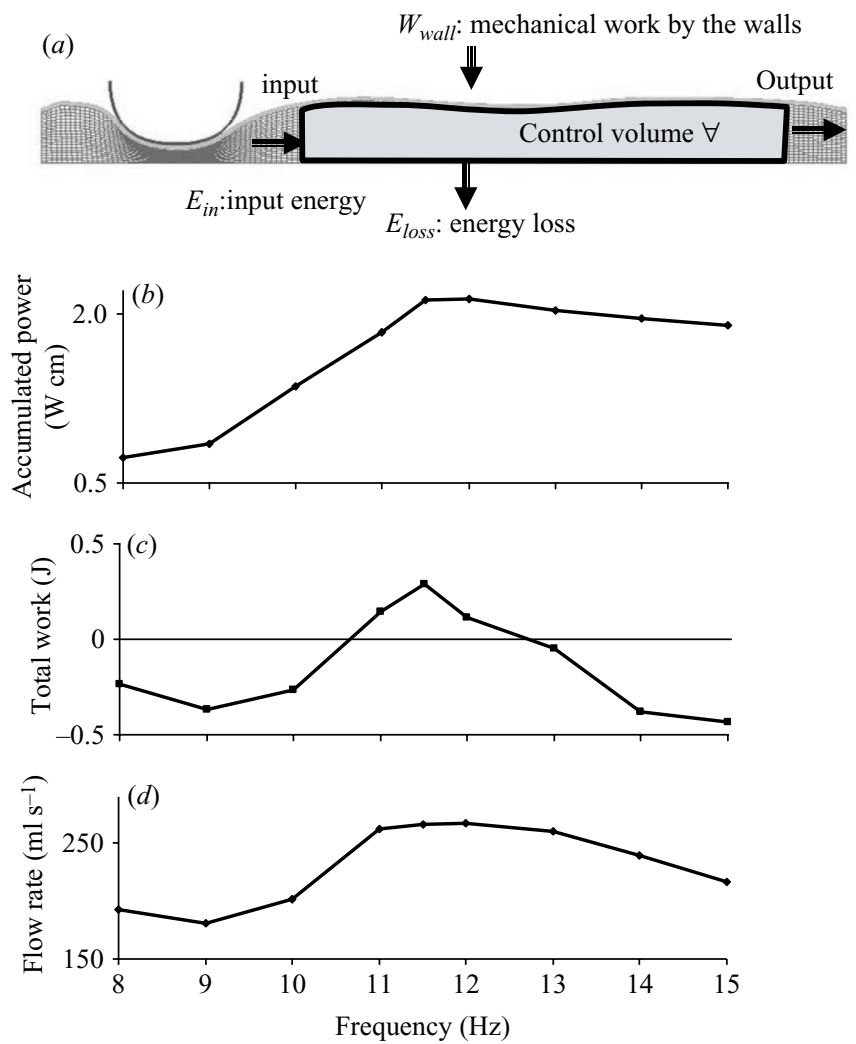

FiguRE 13. Calculation of energy and power along the passive tube: $(a)$ illustration of the control volume, $(b)$ accumulated power converted to fluid energy, $(c)$ total work done on the fluid, compared with bulk flow rate $(d)$ for frequencies $8-15 \mathrm{~Hz}$.

The results are shown in figure $13 b$. It is shown that more power is accumulated for the high flow cases $(11-13 \mathrm{~Hz})$.

For further study of the pumping power transferred to the fluid by the elastic tube, an energy calculation was performed based on the method described in Loumes, Avrahami \& Gharib (2008). The control volume $(\forall)$ selected for the calculation is defined as the long passive region (illustrated in figure $13 a$ ), bounded by the elastic tube and the cross-sections $z=4 \mathrm{~cm}$ (input) and $z=14 \mathrm{~cm}$ (output). In this control volume, the work rate performed by the elastic walls $\dot{W}_{\text {wall }}$ is

$$
\dot{W}_{\text {wall }}=\frac{\partial}{\partial t} \int_{\forall} e \rho \mathrm{d} \forall+\dot{E}_{\text {out }}-\dot{E}_{\text {in }}+\dot{E}_{\text {loss }}
$$

Where $e$ is the total energy ( $e=e_{\text {thermal }}+e_{\text {kinetic }}$ ) in the control volume $\forall, \dot{E}_{\text {loss }}$ is losses due to viscosity and convective heat losses per cycle, and $\dot{E}_{\text {in }}$ and $\dot{E}_{\text {out }}$ are the input and output fluid energy fluxes, respectively.

Assuming periodic conditions, the change in the control-volume internal energy during each cycle is zero. Therefore, the total pump energy added to the fluid at each cycle in the long passive region $W_{\text {pump }}$ can be described as:

$$
W_{\text {pump }}=\int_{T}\left(\dot{W}_{\text {wall }}-\dot{E}_{\text {loss }}\right) \mathrm{d} t=\int_{T}\left(\dot{E}_{\text {out }}-\dot{E}_{\text {in }}\right) \mathrm{d} t .
$$


The input and output energy fluxes through the control surface were calculated from their kinetic and potential components (for further details refer to Loumes et al. 2008):

$$
\begin{aligned}
W_{\text {pump }}= & \int_{T}\left(\iint_{C S}\left[\rho \frac{v^{2}}{2}+p\right] \boldsymbol{v} \cdot \mathrm{d} S\right) \mathrm{d} t \\
= & \int_{T}\left[\frac{\rho}{2} \iint_{\text {output }} v^{3} \mathrm{~d} S-\frac{\rho}{2} \iint_{\text {input }} v^{3} \mathrm{~d} S\right] \mathrm{d} t \\
& +\int_{T}\left[\int_{Q_{t}}^{Q_{t+T}} p_{\text {out }} \mathrm{d} Q_{\text {out }}-\int_{Q_{t}}^{Q_{t+T}} p_{\text {in }} \mathrm{d} Q_{\text {in }}\right] \mathrm{d} t \\
= & \left(E_{k_{\text {out }}}-E_{k_{\text {in }}}\right)+\left(E_{p_{\text {out }}}-E_{p_{\text {in }}}\right)
\end{aligned}
$$

The resulting contribution of the passive tube to the fluid energy for the frequencies $8-15 \mathrm{~Hz}$ is shown in figure $13(c)$. It is distinctly seen in the plot that for the low flow cases, the tube acts as a resistor where energy is decreasing along the tube, while in high flow cases $(11-12 \mathrm{~Hz})$ the total work performed by the passive tube is greater than zero. This result is further evidence that in resonant cases, not only that the passive tube is not a resistor, but it is a pump - the pumping takes place in the 'pumping region' and is a result of the unique wave dynamics in that region.

\section{Discussion}

In this numerical study of impedance pump behavior, the fully coupled fluid and structure flow fields were solved, and a detailed description of the unsteady flow field is given for a wide range of cases. Using numerous simulations of various pinching parameters, several characteristics of the impedance pump are depicted. The effect of pinching location, duty cycle, amplitude and frequency were evaluated and found in agreement with the experimental results (Hickerson et al. 2005).

\subsection{Resonance behaviour}

According to the rise time calculated for the various cases (figure 6), it may be concluded that until the system converges to periodicity, waves originated in several pinching cycles are accumulated in the field and participate in the complex wave dynamics. Thus, the periodic flow field is a result of combinations of waves from several pinching cycles. In the present study, the flow field is affected by waves that travel in the tube for up to 15 cycles. In the experimental results of Borzi \& Propst (2003), rise times were of more than 25 cycles, and in Hickerson (2005) rise times were of the order of 5-10 cycles. The difference between the systems may be attributed to tube material and damping properties.

After reaching periodic conditions, the flow in the pump is very sensitive to the unique arrangement and timing of elastic and flow waves formed in the tube, and thus to pinching frequency. This is the key to the resonant nature of impedance pumping.

Figure 5 shows that the resonance frequencies $(5.9 \mathrm{~Hz}$ and $11.5 \mathrm{~Hz})$ are slightly shifted from the natural frequency of the tube $(6.12 \mathrm{~Hz})$ and its harmonics $(12.23 \mathrm{~Hz})$. According to Crowell (2006), the resonance frequency, $f_{\text {res }}$ (at which the response amplitude of a system to vibrations is maximal), should be slightly smaller than the system's natural frequency, $f_{0}$ (at which it will vibrate as a response to impulse), given by:

$$
f_{\text {res }}=\sqrt{f_{0}^{2}-\frac{1}{2} \mathrm{FWHM}^{2}}
$$


where FWHM is the full width of the resonance peak at half-maximum, which is strongly influenced by the system damping (Crowell 2006). Based on figure 5, the corresponding resonance frequencies for the first and second peaks should be:

$$
\begin{aligned}
& \mathrm{FWHM}_{1} \approx 2 \mathrm{~Hz} \Rightarrow f_{\text {res } 1}=\sqrt{(6.12 \mathrm{~Hz})^{2}-\frac{1}{2}(2 \mathrm{~Hz})^{2}}=5.95 \mathrm{~Hz}, \\
& \mathrm{FWHM}_{2} \approx 4 \mathrm{~Hz} \Rightarrow f_{\text {res } 2}=\sqrt{(12.23 \mathrm{~Hz})^{2}-\frac{1}{2}(4 \mathrm{~Hz})^{2}}=11.9 \mathrm{~Hz},
\end{aligned}
$$

which are strongly correlated with the resonance frequencies obtained. A similar result was found experimentally by Hickerson et al. (2005). However, Manopoulos et al. (2006) found $f_{\text {res }}>f_{0}$ in their one-dimensional numerical model. It may be suggested that other factors, such as radius reduction by the pincher or a lower pressure regime in the tube (due to the high flow produced in the tube) reduce the waves velocity and may also contribute to a frequency shift.

Since in some frequency ranges $(3-5 \mathrm{~Hz})$ frequency test resolution is lower than of the ranges of interest, there might be a slight chance that some insignificant narrow peaks between the tested frequencies are not shown.

\subsection{Pumping mechanism}

The basic mechanism by which a net flow is obtained in the impedance pump is revealed from the simulated flow and pressure fields. Three sequential major factors participate in driving the flow: inertia, volume suction, and pressure gradient.

\section{Inertia}

In each cycle, two flow waves emerge from the upstream region of the tube: $F_{1}$, originated by the pinching and $\mathrm{F}_{2}$ by the restoration of the tube. These flow waves propagate downstream in the region between the pincher and the $\mathrm{VN}$ mainly by inertia (shown by negative flow loops). However, the main mechanism that drives the net flow in the tube is concentrated at the end of the elastic tube downstream of the $\mathrm{VN}$, in the region indicated as 'pumping region' in figure 7.

\section{Volume suction}

The pumping action in the pumping region is mostly a result of pressure wave interaction and reflection. The two pressure waves originated by the pinching $\left(\mathrm{P}_{1}^{+}\right)$and the restoring forces $\left(\mathrm{P}_{2}^{-}\right)$propagate downstream and interact with backward waves $\left(\mathrm{P}_{0}^{+}\right.$and $\left.\mathrm{P}_{\mathrm{C} 1}^{-}\right)$. The forward waves are usually stronger; therefore, after interaction the combined waves $\left(\mathrm{P}_{\mathrm{C} 1}^{+}\right.$and $\left.\mathrm{P}_{\mathrm{C} 2}^{-}\right)$are directed downstream towards the tube end. These combined pressure waves are reflected, reversing signs and directions $\left(\mathrm{P}_{\mathrm{C} !}^{-}\right.$and $\left.\mathrm{P}_{\mathrm{C} 2}^{+}\right)$. All these wave reflections and interactions occur in the pumping region, where the flow loop areas are positive, resulting in a rapid wall expansion (stage 5). This intensive expansion leads to strong volume suction at the VN. Two flow waves of opposite directions merge to fill the void. The average flow rate at the $\mathrm{VN}$ is determined by the absolute momentum of these waves, and is the dominant parameter for the net flow in the pump. Figure 8 shows that for the cases with higher flow rate, the tube expansion is larger and the average flow at the $\mathrm{VN}$ is higher.

\section{Pressure gradient}

The stronger flow wave of the two will continue down the pressure drop after passing the pressure rise. This mechanism will produce positive net flow as long as the combined flow wave has enough momentum to overcome the pressure rise and continue downstream to the outlet. This is the situation in all the cases shown in this 
paper, and therefore the average flow found at the $\mathrm{VN}$ for all the cases is positive and no negative net flow is observed.

\subsection{Energy regime}

Another way to look at the unique fluid and structure dynamics in the impedance pump is through energy conversion. The energy given to the system by the pincher is divided into elastic energy and fluid energy. As shown in figure 12, these two energy forms interchange in turn during the pinching cycle along the long part of the elastic tube (downstream of the pincher).

In the upstream section of this region (just downstream of the pincher), the energy given to the fluid is converted to elastic energy. This is shown in figure 12 as a reduction of the loop areas. The VN is the point where the elastic energy of the tube starts to be converted to fluid energy. The conversion of elastic energy to fluid energy in the pumping region is attributed to the rapid tube expansion followed by volume suction. The net energy carried by the fluid at the outlet during a complete cycle represents the pumping energy of the pump (i.e. pincher and tube). Therefore, the pump's performance is related to the amount of elastic energy converted into fluid energy in the pumping region downstream of the VN. An efficient energy conversion will result in high accumulated energy in the fluid.

According to figure $13(b)$, in all the simulated cases, the accumulated energy along the 'long passive region' is positive, meaning addition of energy to that given to the fluid by the pincher, at the expense of elastic energy from the elastic tube. Thus, the dynamics of the elastic tube enhanced the power of the flow by converting both the fluid and elastic energy given to the system by the pinching, mostly to fluid energy at the outlet. Moreover, according to figure 13(c), in the high flow cases the fluid outlet energy is even higher than the input fluid energy (of the fluid energy downstream of the pincher), meaning that at these cases the added energy is even higher than the losses along the way.

The study presented mainly addresses the flow dynamics in the long passive region (downstream of the pincher). The shorter, upstream section of the tube (the region between the tube inlet and the pincher, i.e. $z=0-1 \mathrm{~cm}$ ) is too short for resonance effects to appear. We assume that if this upstream section was longer, at the appropriate frequencies, it might resonate as well. In such a case, when the complete tube is considered, the two sections from both sides of the pincher would compete with each other. Thus, the total net flow rate in the tube would be determined according to the section with the preferred dynamics at the specific conditions (frequency, amplitude, etc.). According to this hypothesis, such a scenario could lead to negative net flow in the tube - when the dynamics of the upstream section is favour. This hypothesis should be able examined in further work, and if confirmed, it may explain the changes in net flow direction with pinching frequency, location and even amplitude, as reported in other publications (Jung \& Peskin 2001; Borzi \& Propst 2003; Hickerson 2005; Rinderknecht et al. 2005; Manopoulos et al. 2006).

In the present study, no negative flow cases were observed since such a configuration did not occur because of the very short upstream section in the cases studied; in the pinching location test, the short sections did not resonate due to the low frequency used.

The results of this study prove that the main driving mechanism of the flow is attributed to the specific wave dynamics along the passive tube, which is a result only of the excitation at the pincher and reflection at the tube end. Moreover, in resonance 
cases, not only is the long passive tube not a resistor (where power is lost), but it is a pump (a power source). These results reinforce the previous experimental observations (Hickerson et al. 2005; Hickerson \& Gharib 2006) and are in contrast to the mechanism proposed by previous studies (Thomann 1978; Moser et al. 1998; Kenner et al. 2000; Manopoulos et al. 2006) relying on fluid inertia or asymmetry in energy losses.

According to this work, pumping could be achieved with any impedance mismatch at the tube boundary. The outlet configuration does not necessarily need to be a tube or have different impedance. A reflection site can be a ring, a bifurcation or a local geometry deformation. Moreover, pumping can be achieved even if the tube is not connected to any other tubing, as long as it has wave reflection sites at its ends and an active element producing frequent pressure waves at an asymmetric location. The present numerical model simulates an elastic tube connected to two equal-pressure reservoirs at its ends using rigid connections, similar to the experimental model conducted by Hickerson et al. (2005), where the tube was connected to two connected reservoirs. Therefore, it included only an elastic tube with complete reflection sites at its ends, with zero pressure at both ends. Yet, even in that configuration, resonance wave pumping was obtained.

The results presented here are of great significance when designing and optimizing impedance pumps. Moreover, it was recently shown that the embryonic heart utilizes impedance pumping to drive circulation in early embryonic stages prior to valve formation (Forouhar et al. 2006). The model presented may be helpful for the investigation of wave dynamics in the embryonic heart and in other applications of the cardiovascular system.

This research was supported by the Joseph Drown Foundation and by a research collaboration grant with the Division of Materials Technology at Nanyang Technological University, Singapore. The authors wish to thank Professor Moshe Rosenfeld from Tel Aviv University for his advice.

\section{REFERENCES}

ADINA R\&D, Inc. 2005 Theory and modeling guide, Volume I: ADINA Solids \& Structures. Watertown, MA.

Auerbach, D., Moehring, W. \& Moser, M. 2004 An analytic approach to the Liebau problem of valveless pumping. Cardiovascular Engng 4, 201.

Bathe, K. J. 1996 Finite Element Procedures. Prentice-Hall.

Borzi, A. \& Propst, G. 2003 Numerical investigation of the Liebau phenomenon. Z. Angew. Math. Phys. 54, 1050-1072.

Crowell, B. 2006 Vibrations and Waves. Fullerton, California, Light and Matter series.

Forouhar, A. S., Liebling, M., Hickerson, A., Nasiraei-Moghaddam, A., Tsai, H. J., Hove, J. R., Fraser, S. E., Dickinson, M. E. \& Gharib, M. 2006 The embryonic vertebrate heart tube is a dynamic suction pump. Science 312, 751-753.

Hickerson, A. I. 2005 An experimental analysis of the characteristic behavior of an impedance pump. PhD thesis, Caltech.

Hickerson, A. I. \& GHARIB, M. 2003 Flow characterization of a valveless impedance driven pump. Annual meeting of the APS Division of Fluid Dynamics, East Rutherford, NJ, November 23-25, 2003.

Hickerson, A. I. \& Gharib, M. 2006 On the resonance of a pliant tube as a mechanism for valveless pumping. J. Fluid Mech. 555, 141-148.

Hickerson, A. I., RinderkneCht, D. \& GHARIB, M. 2005 Experimental study of the behavior of a valveless impedance pump. Exps. Fluids 38, 534-540; and correction 39, 787-787.

Jung, E. \& Peskin, C. 2001 Two-dimensional simulations of valveless pumping using the immersed boundary method. SIAM J. Sci. Comput. 23, 19-45. 
Kenner, T., Moser, M., Tanev, I. \& Ono, K. 2000 The Liebau-Effect or on the optimal use of energy for the circulation of blood. Scripta med 73, 9-14.

Liebau, G. 1954 Uber Ein Ventilloses Pumpprinzip. Naturwissenschaften 41, 327-327.

Liebau, G. 1955 Prinzipien Kombinierter Ventilloser Pumpen, Abgeleitet Vom Menschlichen Blutkreislauf. Naturwissenschaften 42, 339-339.

LiebaU, G. 1963 Uber Die Funktionelle Bedeutung Der Venenklappen. Z. Kreislaufforschung 52, $419-424$.

Loumes, L., Avrahami, I. \& Gharib, M. 2008 Resonant pumping in a multilayer impedance pump. Phys. Fluids 20, 023103.

Manopoulos, C. G., Mathioulakis, D. S. \& Tsangaris, S. G. 2006 One-dimensional model of valveless pumping in a closed loop and a numerical solution. Phys. Fluids 18.

Moser, M., J. Hunang, J. W., Schwarz, G. S., Kenner, T. \& Noordergraaf, A. 1998 Impedance defined flow : generalization of William Harvey's concept of the circulation - 370 years later Intl J. Cardiovasc. Med. Sci. 1, 205-211.

Nichols, W. W. \& O'Rourke, M. F. 1998 McDonald's Blood Flow in Arteries. London, Arnold.

OtTeSEN, J. T. 2003 Valveless pumping in a fluid-filled closed elastic tube-system: one-dimensional theory with experimental validation. J. Math. Biol. 46, 309-332.

Rath, H. J. \& Teipel, I. 1978 Pumping effect In valveless elastic tubes. Z. Angew. Math. Phys. 29, $123-133$.

Rinderknecht, D., Hickerson, A. I. \& Gharib, M. 2005 A valveless micro impedance pump driven by electromagnetic actuation. J. Micromech. Microengng 15, 861-866.

Rosenfeld, M. \& KWAK, D. 1991 Time-dependent solutions of viscous incompressible flows in moving co-ordinates. Intl J. Numer. Meth. Fluids 13, 1311-1328.

Rugonyi, S. \& Bathe, K. J. 2001 On finite element analysis of fluid flows fully coupled with structural interactions. Cmes-Computer Model. Engng Sci. 2, 195-212.

Thomann, H. 1978 Simple pumping mechanism in a valveless tube. Z. Angew. Math. Phys. 29, 169-177.

Zamir, M. 2000 The Physics of Pulsatile Flow. Springer. 